\title{
A global model for joint lemmatization and part-of-speech prediction
}

\author{
Kristina Toutanova \\ Microsoft Research \\ Redmond, WA 98052 \\ kristoutemicrosoft.com
}

\author{
Colin Cherry \\ Microsoft Research \\ Redmond, WA 98052 \\ colinc@microsoft.com
}

\begin{abstract}
We present a global joint model for lemmatization and part-of-speech prediction. Using only morphological lexicons and unlabeled data, we learn a partiallysupervised part-of-speech tagger and a lemmatizer which are combined using features on a dynamically linked dependency structure of words. We evaluate our model on English, Bulgarian, Czech, and Slovene, and demonstrate substantial improvements over both a direct transduction approach to lemmatization and a pipelined approach, which predicts part-of-speech tags before lemmatization.
\end{abstract}

\section{Introduction}

The traditional problem of morphological analysis is, given a word form, to predict the set of all of its possible morphological analyses. A morphological analysis consists of a part-of-speech tag (POS), possibly other morphological features, and a lemma (basic form) corresponding to this tag and features combination (see Table 1 for examples). We address this problem in the setting where we are given a morphological dictionary for training, and can additionally make use of un-annotated text in the language. We present a new machine learning model for this task setting.

In addition to the morphological analysis task we are interested in performance on two subtasks: tag-set prediction (predicting the set of possible tags of words) and lemmatization (predicting the set of possible lemmas). The result of these subtasks is directly useful for some applications. ${ }^{1}$ If we are interested in the results of each of these two

\footnotetext{
${ }^{1}$ Tag sets are useful, for example, as a basis of sparsityreducing features for text labeling tasks; lemmatization is useful for information retrieval and machine translation from a morphologically rich to a morphologically poor language, where full analysis may not be important.
}

subtasks in isolation, we might build independent solutions which ignore the other subtask.

In this paper, we show that there are strong dependencies between the two subtasks and we can improve performance on both by sharing information between them. We present a joint model for these two subtasks: it is joint not only in that it performs both tasks simultaneously, sharing information, but also in that it reasons about multiple words jointly. It uses component tag-set and lemmatization models and combines their predictions while incorporating joint features in a loglinear model, defined on a dynamically linked dependency structure of words.

The model is formalized in Section 5 and evaluated in Section 6. We report results on English, Bulgarian, Slovene, and Czech and show that joint modeling reduces the lemmatization error by up to $19 \%$, the tag-prediction error by up to $26 \%$ and the error on the complete morphological analysis task by up to $22.6 \%$.

\section{Task formalization}

The main task that we would like to solve is as follows: given a lexicon $L$ which contains all morphological analyses for a set of words $\left\{w_{1}, \ldots, w_{n}\right\}$, learn to predict all morphological analyses for other words which are outside of $L$. In addition to the lexicon, we are allowed to make use of unannotated text $\mathrm{T}$ in the language. We will predict morphological analyses for words which occur in $T$. Note that the task is defined on word types and not on words in context.

A morphological analysis of a word $w$ consists of a (possibly structured) POS tag $t$, together with one or several lemmas, which are the possible basic forms of $w$ when it has tag $t$. As an example, Table 1 illustrates the morphological analyses of several words taken from the CELEX lexical database of English (Baayen et al., 1995) and the Multext-East lexicon of Bulgarian (Erjavec, 2004). The Bulgarian words are transcribed in 


\begin{tabular}{|c|c|c|c|}
\hline $\begin{array}{l}\text { Word Forms } \\
\text { tell }\end{array}$ & $\begin{array}{l}\text { Morphological Analyses } \\
\text { verb base (VB), tell }\end{array}$ & $\begin{array}{l}\text { Tags } \\
\text { VB }\end{array}$ & $\begin{array}{r}\text { Lemmas } \\
\text { tell }\end{array}$ \\
\hline told & $\begin{array}{l}\text { verb past tense (VBD), tell } \\
\text { verb past participle (VBN), tell }\end{array}$ & VBD, VBN & tell \\
\hline tells & verb present 3rd person sing (VBZ), tell & VBZ & tell \\
\hline telling & $\begin{array}{l}\text { verb present continuous }(\mathrm{VBG}), \text { tell } \\
\text { adjective }(\mathrm{JJ}) \text {, telling }\end{array}$ & VBG,JJ & $\begin{array}{r}\text { tell } \\
\text { telling }\end{array}$ \\
\hline izpravena & $\begin{array}{l}\text { adjective fem sing indef (A-FS-N), izpraven } \\
\text { verb main part past sing fem pass indef (VMPS-SFP-N), izpravia }\end{array}$ & $\begin{array}{l}\text { A-FS-N } \\
\text { VMPS-SFP-N }\end{array}$ & $\begin{array}{c}\text { izpraven } \\
\text { izpravia }\end{array}$ \\
\hline izpraviha & verb main indicative 3rd person plural (VMIA3P), izpravia & VMIA3P & izpravia \\
\hline
\end{tabular}

Table 1: Examples of morphological analyses of words in English and Bulgarian.

Latin characters. Here by "POS tags" we mean both simple main pos-tags such as noun or verb, and detailed tags which include grammatical features, such as VBZ for English indicating present tense third person singular verb and A-FS-N for Bulgarian indicating a feminine singular adjective in indefinite form. In this work we predict only main POS tags for the Multext-East languages, as detailed tags were less useful for lemmatization.

Since the predicted elements are sets, we use precision, recall, and F-measure (F1) to evaluate performance. The two subtasks, tag-set prediction and lemmatization are also evaluated in this way. Table 1 shows the correct tag-sets and lemmas for each of the example words in separate columns. Our task setting differs from most work on lemmatization which uses either no or a complete rootlist (Wicentowski, 2002; Dreyer et al., 2008). ${ }^{2}$ We can use all forms occurring in the unlabeled text $\mathrm{T}$ but there are no guarantees about the coverage of the target lemmas or the number of noise words which may occur in $T$ (see Table 2 for data statistics). Our setting is thus more realistic since it is what one would have in a real application scenario.

\section{Related work}

In work on morphological analysis using machine learning, the task is rarely addressed in the form described above. Some exceptions are the work (Bosch and Daelemans, 1999) which presents a model for segmenting, stemming, and tagging words in Dutch, and requires the prediction of all possible analyses, and (Antal van den Bosch and Soudi, 2007) which similarly requires the prediction of all morpho-syntactically annotated segmentations of words for Arabic. As opposed to

\footnotetext{
${ }^{2}$ These settings refer to the availability of a set of word forms which are possible lemmas; in the no rootlist setting, no other word forms in the language are given in addition to the forms in the training set; in the complete rootlist setting, a set of word forms which consists of exactly all correct lemmas for the words in the test set is given.
}

our work, these approaches do not make use of unlabeled data and make predictions for each word type in isolation.

In machine learning work on lemmatization for highly inflective languages, it is most often assumed that a word form and a POS tag are given, and the task is to predict the set of corresponding lemma(s) (Mooney and Califf, 1995; Clark, 2002; Wicentowski, 2002; Erjavec and Džeroski, 2004; Dreyer et al., 2008). In our task setting, we do not assume the availability of gold-standard POS tags. As a component model, we use a lemmatizing string transducer which is related to these approaches and draws on previous work in this and related string transduction areas. Our transducer is described in detail in Section 4.1.

Another related line of work approaches the disambiguation problem directly, where the task is to predict the correct analysis of word-forms in context (in sentences), and not all possible analyses. In such work it is often assumed that the correct POS tags can be predicted with high accuracy using labeled POS-disambiguated sentences (Erjavec and Džeroski, 2004; Habash and Rambow, 2005). A notable exception is the work of (Adler et al., 2008), which uses unlabeled data and a morphological analyzer to learn a semi-supervised HMM model for disambiguation in context, and also guesses analyses for unknown words using a guesser of likely POS-tags. It is most closely related to our work, but does not attempt to predict all possible analyses, and does not have to tackle a complex string transduction problem for lemmatization since segmentation is mostly sufficient for the focus language of that study (Hebrew).

The idea of solving two related tasks jointly to improve performance on both has been successful for other pairs of tasks (e.g., (Andrew et al., 2004)). Doing joint inference instead of taking a pipeline approach has also been shown useful for other problems (e.g., (Finkel et al., 2006; Cohen and Smith, 2007)). 


\section{Component models}

We use two component models as the basis of addressing the task: one is a partially-supervised POS tagger which is trained using $L$ and the unlabeled text $\mathrm{T}$; the other is a lemmatizing transducer which is trained from $L$ and can use T. The transducer can optionally be given input POS tags in training and testing, which can inform the lemmatization. The tagger is described in Section 4.2 and the transducer is described in Section 4.1.

In a pipeline approach to combining the tagging and lemmatization components, we first predict a set of tags for each word using the tagger, and then ask the lemmatizer to predict one lemma for each of the possible tags. In a direct transduction approach to the lemmatization subtask, we train the lemmatizer without access to tags and ask it to predict a single lemma for each word in testing. Our joint model, described in Section 5, is defined in a re-ranking framework, and can choose from among $k$-best predictions of tag-sets and lemmas generated from the component tagger and lemmatizer models.

\subsection{Morphological analyser}

We employ a discriminative character transducer as a component morphological analyzer. The input to the transducer is an inflected word (the source) and possibly an estimated part-of-speech; the output is the lemma of the word (the target). The transducer is similar to the one described by $\mathrm{Ji}$ ampojamarn et al. (2008) for letter-to-phoneme conversion, but extended to allow for whole-word features on both the input and the output. The core of our engine is the dynamic programming algorithm for monotone phrasal decoding (Zens and Ney, 2004). The main feature of this algorithm is its capability to transduce many consecutive characters with a single operation; the same algorithm is employed to tag subsequences in semi-Markov CRFs (Sarawagi and Cohen, 2004).

We employ three main categories of features: context, transition, and vocabulary (rootlist) features. The first two are described in detail by Jiampojamarn et al. (2008), while the final is novel to this work. Context features are centered around a transduction operation such as $e s \rightarrow e$, as employed in gives $\rightarrow$ give. Context features include an indicator for the operation itself, conjoined with indicators for all $n$-grams of source context within a fixed window of the operation. We also employ a copy feature that indicates if the operation simply copies the source character, such as $e \rightarrow e$. Transition features are our Markov, or $n$-gram features on transduction operations. Vocabulary features are defined on complete target words, according to the frequency of said word in a provided unlabeled text $\mathrm{T}$. We have chosen to bin frequencies; experiments on a development set suggested that two indicators are sufficient: the first fires for any word that occurred fewer than five times, while a second also fires for those words that did not occur at all. By encoding our vocabulary in a trie and adding the trie index to the target context tracked by our dynamic programming chart, we can efficiently track these frequencies during transduction.

We incorporate the source part-of-speech tag by appending it to each feature, thus the context feature $e s \rightarrow e$ may become $e s \rightarrow e$, VBZ. To enable communication between the various parts-ofspeech, a universal set of unannotated features also fires, regardless of the part-of-speech, acting as a back-off model of how words in general behave during stemming.

Linear weights are assigned to each of the transducer's features using an averaged perceptron for structure prediction (Collins, 2002). Note that our features are defined in terms of the operations employed during transduction, therefore to create gold-standard feature vectors, we require not only target outputs, but also derivations to produce those outputs. We employ a deterministic heuristic to create these derivations; given a goldstandard source-target pair, we construct a derivation that uses only trivial copy operations until the first character mismatch. The remainder of the transduction is performed with a single multicharacter replacement. For example, the derivation for living $\rightarrow$ live would be $l \rightarrow l, i \rightarrow i$, $v \rightarrow v$, ing $\rightarrow e$. For languages with morphologies affecting more than just the suffix, one can either develop a more complex heuristic, or determine the derivations using a separate aligner such as that of Ristad and Yianilos (1998).

\subsection{Tag-set prediction model}

The tag-set model uses a training lexicon $L$ and unlabeled text $T$ to learn to predict sets of tags for words. It is based on the semi-supervised tagging model of (Toutanova and Johnson, 2008). It has two sub-models: one is an ambiguity class 
or a tag-set model, which can assign probabilities for possible sets of tags of words $P_{T S M}(t s \mid w)$ and the other is a word context model, which can assign probabilities $P_{C M}\left(\right.$ context $\left.s_{w} \mid w, t s\right)$ to all contexts of occurrence of word $w$ in an unlabeled text T. The word-context model is Bayesian and utilizes a sparse Dirichlet prior on the distributions of tags given words. In addition, it uses information on a four word context of occurrences of $w$ in the unlabeled text.

Note that the (Toutanova and Johnson, 2008) model is a tagger that assigns tags to occurrences of words in the text, whereas we only need to predict sets of possible tags for word types, such as the set $\{\mathrm{VBD}, \mathrm{VBN}\}$ for the word told. Their component sub-model $P_{T S M}$ predicts sets of tags and it is possible to use it on its own, but by also using the context model we can take into account information from the context of occurrence of words and compute probabilities of tag-sets given the observed occurrences in T. The two are combined to make a prediction for a tag-set of a test word $w$, given unlabeled text $\mathrm{T}$, using Bayes rule: $p(t s \mid w) \propto P_{T S M}(t s \mid w) P_{C M}\left(\right.$ contexts $\left._{w} \mid w, t s\right)$.

We use a direct re-implementation of the wordcontext model, using variational inference following (Toutanova and Johnson, 2008). For the tagset sub-model, we employ a more sophisticated approach. First, we learn a log-linear classifier instead of a Naive Bayes model, and second, we use features derived from related words appearing in T. The possible classes predicted by the classifier are as many as the observed tag-sets in $L$. The sparsity is relieved by adding features for individual tags $t$ which get shared across tag-sets containing $t$.

There are two types of features in the model: (i) word-internal features: word suffixes, capitalization, existence of hyphen, and word prefixes (such features were also used in (Toutanova and Johnson, 2008)), and (ii) features based on related words. These latter features are inspired by (Cucerzan and Yarowsky, 2000) and are defined as follows: for a word $w$ such as telling, there is an indicator feature for every combination of two suffixes $\alpha$ and $\beta$, such that there is a prefix $p$ where telling $=p \alpha$ and $p \beta$ exists in T. For example, if the word tells is found in $\mathrm{T}$, there would be a feature for the suffixes $\alpha=i n g, \beta=s$ that fires. The suffixes are defined as all character suffixes up to length three which occur with at least 100 words.

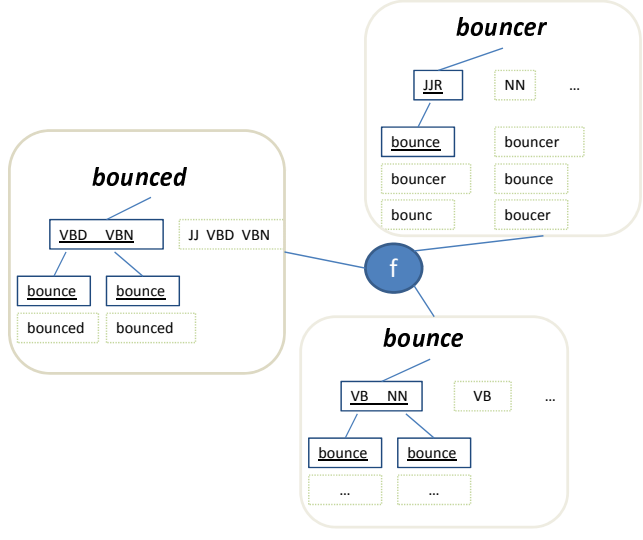

Figure 1: A small subset of the graphical model. The tag-sets and lemmas active in the illustrated assignment are shown in bold. The extent of joint features firing for the lemma bounce is shown as a factor indicated by the blue circle and connected to the assignments of the three words.

\section{A global joint model for morphological analysis}

The idea of this model is to jointly predict the set of possible tags and lemmas of words. In addition to modeling dependencies between the tags and lemmas of a single word, we incorporate dependencies between the predictions for multiple words. The dependencies among words are determined dynamically. Intuitively, if two words have the same lemma, their tag-sets are dependent. For example, imagine that we need to determine the tag-set and lemmas of the word bouncer. The tagset model may guess that the word is an adjective in comparative form, because of its suffix, and because its occurrences in $\mathrm{T}$ might not strongly indicate that it is a noun. The lemmatizer can then lemmatize the word like an adjective and come up with bounce as a lemma. If the tag-set model is fairly certain that bounce is not an adjective, but is a verb or a noun, a joint model which looks simultaneously at the tags and lemmas of bouncer and bounce will detect a problem with this assignments and will be able to correct the tagging and lemmatization error for bouncer.

The main source of information our joint model uses is information about the assignments of all words that have the same lemma $l$. If the tag-set model is better able to predict the tags of some of these words, the information can propagate to the other words. If some of them are lemmatized correctly, the model can be pushed to lemmatize the others correctly as well. Since the lemmas of test words are not given, the dependencies between as- 
signments of words are determined dynamically by the currently chosen set of lemmas.

As an example, Figure 1 shows three sample English words and their possible tag-sets and lemmas determined by the component models. It also illustrates the dependencies between the variables induced by the features of our model active for the current (incorrect) assignment.

\subsection{Formal model description}

Given a set of test words $w_{1}, \ldots w_{n}$ and additional word forms occurring in unlabeled data $\mathrm{T}$, we derive an extended set of words $w_{1}, \ldots, w_{m}$ which contains the original test words and additional related words, which can provide useful information about the test words. For example, if bouncer is a test word and bounce and bounced occur in T these two words can be added to the set of test words because they can contribute to the classification of bouncer. The algorithm for selecting related words is simple: we add any word for which the pipelined model predicts a lemma which is also predicted as one of the top $k$ lemmas for a word from the test set.

We define a joint model over tag-sets and lemmas for all words in the extended set, using features defined on a dynamically linked structure of words and their assigned analyses. It is a reranking model because the tag-sets and possible lemmas are limited to the top $k$ options provided by the pipelined model. ${ }^{3}$ Our model is defined on a very large set of variables, each of which can take a large set of values. For example, for a test set of size about 4,000 words for Slovene an additional about 9,000 words from $T$ were added to the extended set. Each of these words has a corresponding variable which indicates its tag-set and lemma assignment. The possible assignments range over all combinations available from the tagging and lemmatizer component models; using the top three tag-sets per word and top three lemmas per tag gives an average of around 11.2 possible assignments per word. This is because the tagsets have about 1.2 tags on average and we need to choose a lemma for each. While it is not the case that all variables are connected to each other by features, the connectivity structure can be complex.

More formally, let $t s_{i}^{j}$ denote possible tag-sets

\footnotetext{
${ }^{3}$ We used top three tag-sets and top three lemmas for each tag for training.
}

for word $w_{i}$, for $j=1 \ldots k$. Also, let $l_{i}(t)^{j}$ denote the top lemmas for word $w_{i}$ given tag $t$. An assignment of a tag-set and lemmas to a word $w_{i}$ consists of a choice of a tag-set, $t s_{i}$ (one of the possible $k$ tag-sets for the word) and, for each tag $t$ in the chosen tag-set, a choice of a lemma out of the possible lemmas for that tag and word. For brevity, we denote such joint assignment by $t l_{i}$. As a concrete example, in Figure 1, we can see the current assignments for three words: the assigned tag-sets are shown underlined and in bolded boxes (e.g., for bounced, the tag-set $\{\mathrm{VBD}, \mathrm{VBN}\}$ is chosen; for both tags, the lemma bounce is assigned). Other possible tag-sets and other possible lemmas for each chosen tag are shown in greyed boxes.

Our joint model defines a distribution over assignments to all words $w_{1}, \ldots, w_{m}$. The form of the model is as follows:

$$
P\left(t l_{1}, \ldots, t l_{m}\right)=\frac{e^{F\left(t l_{1}, \ldots, t l_{m}\right)^{\prime} \theta}}{\sum_{t l_{1}^{\prime}, \ldots, t l_{m}^{\prime}} e^{F\left(t l_{1}^{\prime}, \ldots, t l_{m}^{\prime}\right)^{\prime} \theta}}
$$

Here $\mathrm{F}$ denotes the vector of features defined over an assignment for all words in the set and $\theta$ is a vector of parameters for the features. Next we detail the types of features used.

Word-local features. The aim of such features is to look at the set of all tags assigned to a word together with all lemmas and capture coarse-grained dependencies at this level. These features introduce joint dependencies between the tags and lemmas of a word, but they are still local to the assignment of single words. One such feature is the number of distinct lemmas assigned across the different tags in the assigned tag-set. Another such feature is the above joined with the identity of the tag-set. For example, if a word's tag-set is $\{\mathrm{VBD}, \mathrm{VBN}\}$, it will likely have the same lemma for both tags and the number of distinct lemmas will be one (e.g., the word bounced), whereas if it has the tags VBG, JJ the lemmas will be distinct for the two tags (e.g. telling). In this class of features are also the log-probabilities from the tag-set and lemmatizer models.

Non-local features. Our non-local features look, for every lemma $l$, at all words which have that lemma as the lemma for at least one of their assigned tags, and derive several predicates on the joint assignment to these words. For example, using our word graph in the figure, the lemma bounce is assigned to bounced for tags $\mathrm{VBD}$ and $\mathrm{VBN}$, to bounce for tags $\mathrm{VB}$ and $\mathrm{NN}$, and to bouncer for tag JJR. One feature looks at the combination of tags corresponding to the differ- 
ent forms of the lemma. In this case this would be [JJR,NN+VB-lem, VBD+VBN]. The feature also indicates any word which is exactly equal to the lemma with lem as shown for the NN and VB tags corresponding to bounce. Our model learns a negative weight for this feature, because the lemma of a word with tag JJR is most often a word with at least one tag equal to JJ. A variant of this feature also appends the final character of each word, like this: [JJR+r, NN+VB+e-lem, $\mathrm{VBD}+\mathrm{VBN}-$ d]. This variant was helpful for the Slavic languages because when using only main POS tags, the granularity of the feature is too coarse. Another feature simply counts the number of distinct words having the same lemma, encouraging reusing the same lemma for different words. An additional feature fires for every distinct lemma, in effect counting the number of assigned lemmas.

\subsection{Training and inference}

Since the model is defined to re-rank candidates from other component models, we need two different training sets: one for training the component models, and another for training the joint model features. This is because otherwise the accuracy of the component models would be overestimated by the joint model. Therefore, we train the component models on the training lexicons LTrain and select their hyperparameters on the LDev lexicons. We then train the joint model on the LDev lexicons and evaluate it on the LTest lexicons. When applying models to the LTest set, the component models are first retrained on the union of LTrain and LDev so that all models can use the same amount of training data, without giving unfair advantage to the joint model. Such set-up is also used for other re-ranking models (Collins, 2000).

For training the joint model, we maximize the log-likelihood of the correct assignment to the words in LDev, marginalizing over the assignments of other related words added to the graphical model. We compute the gradient approximately by computing expectations of features given the observed assignments and marginal expectations of features. For computing these expectations we use Gibbs sampling to sample complete assignments to all words in the graph. ${ }^{4} \mathrm{We}$

\footnotetext{
${ }^{4}$ We start the Gibbs sampler by the assignments found by the pipeline method and then use an annealing schedule to find a neighborhood of high-likelihood assignments, before taking about 10 complete samples from the graph to compute expectations.
}

use gradient descent with a small learning rate, selected to optimize the accuracy on the LDev set. For finding a most likely assignment at test time, we use the sampling procedure, this time using a slower annealing schedule before taking a single sample to output as a guessed answer.

For the Gibbs sampler, we need to sample an assignment for each word in turn, given the current assignments of all other words. Let us denote the current assignment to all words except $w_{i}$ as $\mathbf{t l}^{-i}$. The conditional probability of an assignment $t l_{i}$ for word $w_{i}$ is given by:

$$
P\left(t l_{i} \mid \mathbf{t} \mathbf{l}^{-i}\right)=\frac{e^{F\left(t l_{i}, \mathbf{t l}^{-i}\right)^{\prime} \theta}}{\sum_{t l_{i}^{\prime}} e^{F\left(t l_{i}^{\prime}, \mathbf{t l}^{-i}\right)^{\prime} \theta}}
$$

The summation in the denominator is over all possible assignments for word $w_{i}$. To compute these quantities we need to consider only the features involving the current word. Because of the nature of the features in our model, it is possible to isolate separate connected components which do not share features for any assignment. If two words do not share lemmas for any of their possible assignments, they will be in separate components. Block sampling within a component could be used if the component is relatively small; however, for the common case where there are five or more words in a fully connected component approximate inference is necessary.

\section{Experiments}

\subsection{Data}

We use datasets for four languages: English, Bulgarian, Slovene, and Czech. For each of the languages, we need a lexicon with morphological analyses $L$ and unlabeled text.

For English we derive the lexicon from CELEX (Baayen et al., 1995), and for the other languages we use the Multext-East resources (Erjavec, 2004). For English we use only open-class words (nouns, verbs, adjectives, and adverbs), and for the other languages we use words of all classes. The unlabeled data for English we use is the union of the Penn Treebank tagged WSJ data (Marcus et al., 1993) and the BLLIP corpus. ${ }^{5}$ For the rest of the languages we use only the text of George Orwell's novel 1984, which is provided in morphologically disambiguated form as part of MultextEast (but we don't use the annotations). Table 2

\footnotetext{
${ }^{5}$ The BLLIP corpus contains approximately 30 million words of automatically parsed WSJ data. We used these corpora as plain text, without the annotations.
} 


\begin{tabular}{|l|ccc|ccc|ccc|c|}
\hline Lang & \multicolumn{3}{|c}{ LTrain } & \multicolumn{3}{c|}{ LDev } & \multicolumn{3}{c|}{ LTest } & Text \\
& ws & tl & nf & ws & tl & nf & ws & tl & nf & \\
\hline Eng & 5.2 & 1.5 & 0.3 & 7.4 & 1.4 & 0.8 & 7.4 & 1.4 & 0.8 & 320 \\
Bgr & 6.9 & 1.2 & 40.8 & 3.8 & 1.1 & 53.6 & 3.8 & 1.1 & 52.8 & 16.3 \\
Slv & 7.5 & 1.2 & 38.3 & 4.2 & 1.2 & 49.1 & 4.2 & 1.2 & 49.8 & 17.8 \\
Cz & 7.9 & 1.1 & 32.8 & 4.5 & 1.1 & 43.2 & 4.5 & 1.1 & 43.0 & 19.1 \\
\hline
\end{tabular}

Table 2: Data sets used in experiments. The number of word types (ws) is shown approximately in thousands. Also shown are average number of complete analyses (tl) and percent target lemmas not found in the unlabeled text (nf).

details statistics about the data set sizes for different languages.

We use three different lexicons for each language: one for training (LTrain), one for development (LDev), and one for testing (LTest). The global model weights are trained on the development set as described in section 5.2. The lexicons are derived such that very frequent words are likely to be in the training lexicon and less frequent words in the dev and test lexicons, to simulate a natural process of lexicon construction. The English lexicons were constructed as follows: starting with the full CELEX dictionary and the text of the Penn Treebank corpus, take all word forms appearing in the first 2000 sentences (and are found in CELEX) to form the training lexicon, and then take all other words occurring in the corpus and split them equally between the development and test lexicons (every second word is placed in the test set, in the order of first occurrence in the corpus). For the rest of the languages, the same procedure is applied, starting with the full Multext-East lexicons and the text of the novel 1984. Note that while it is not possible for training words to be included in the other lexicons, it is possible for different forms of the same lemma to be in different lexicons. The size of the training lexicons is relatively small and we believe this is a realistic scenario for application of such models. In Table 2 we can see the number of words in each lexicon and the unlabeled corpora (by type), the average number of tag-lemma combinations per word, ${ }^{6}$ as well as the percentage of word lemmas which do not occur in the unlabeled text. For English, the large majority of target lemmas are available in T (with only $0.8 \%$ missing), whereas for the Multext-East languages around 40 to $50 \%$ of the target lemmas are not found in $\mathrm{T}$; this partly explains the lower performance on these languages.

\footnotetext{
${ }^{6}$ The tags are main tags for the Multext-East languages and detailed tags for English.
}

\begin{tabular}{|l|l|c|c|c|}
\hline Language & Tag Model & Tag & Lem & T+L \\
English & none & - & 94.0 & - \\
& full & 89.9 & 95.3 & 88.9 \\
& no unlab data & 80.0 & 94.1 & 78.3 \\
Bulgarian & none & - & 73.2 & - \\
& full & 87.9 & 79.9 & 75.3 \\
& no unlab data & 80.2 & 76.3 & 70.4 \\
\hline
\end{tabular}

Table 3: Development set results using different tag-set models and pipelined prediction.

\subsection{Evaluation of direct and pipelined models for lemmatization}

As a first experiment which motivates our joint modeling approach, we present a comparison on lemmatization performance in two settings: (i) when no tags are used in training or testing by the transducer, and (ii) when correct tags are used in training and tags predicted by the tagging model are used in testing. In this section, we report performance on English and Bulgarian only. Comparable performance on the other Multext-East languages is shown in Section 6.

Results are presented in Table 3. The experiments are performed using LTrain for training and LDev for testing. We evaluate the models on tagset F-measure (Tag), lemma-set F-measure(Lem) and complete analysis F-measure $(\mathrm{T}+\mathrm{L})$. We show the performance on lemmatization when tags are not predicted (Tag Model is none), and when tags are predicted by the tag-set model. We can see that on both languages lemmatization is significantly improved when a latent tag-set variable is used as a basis for prediction: the relative error reduction in Lem F-measure is $21.7 \%$ for English and 25\% for Bulgarian. For Bulgarian and the other Slavic languages we predicted only main POS tags, because this resulted in better lemmatization performance.

It is also interesting to evaluate the contribution of the unlabeled data $T$ to the performance of the tag-set model. This can be achieved by removing the word-context sub-model of the tagger and also removing related word features. The results achieved in this setting for English and Bulgarian are shown in the rows labeled "no unlab data". We can see that the tag-set F-measure of such models is reduced by 8 to 9 points and the lemmatization F-measure is similarly reduced. Thus a large portion of the positive impact tagging has on lemmatization is due to the ability of tagging models to exploit unlabeled data.

The results of this experiment show there are strong dependencies between the tagging and 
lemmatization subtasks, which a joint model could exploit.

\subsection{Evaluation of joint models}

Since our joint model re-ranks candidates produced by the component tagger and lemmatizer, there is an upper bound on the achievable performance. We report these upper bounds for the four languages in Table 4 , at the rows which list $m$-best oracle under Model. The oracle is computed using five-best tag-set predictions and three-best lemma predictions per tag. We can see that the oracle performance on tag F-measure is quite high for all languages, but the performance on lemmatization and the complete task is close to only 90 percent for the Slavic languages. As a second oracle we also report the perfect tag oracle, which selects the lemmas determined by the transducer using the correct part-of-speech tags. This shows how well we could do if we made the tagging model perfect without changing the lemmatizer. For the Slavic languages this is quite a bit lower than the $m$-best oracles, showing that the majority of errors of the pipelined approach cannot be fixed by simply improving the tagging model. Our global model has the potential to improve lemma assignments even given correct tags, by sharing information among multiple words.

The actual achieved performance for three different models is also shown. For comparison, the lemmatization performance of the direct transduction approach which makes no use of tags is also shown. The pipelined models select onebest tag-set predictions from the tagging model, and the 1-best lemmas for each tag, like the models used in Section 6.2. The model name local FS denotes a joint log-linear model which has only word-internal features. Even with only word-internal features, performance is improved for most languages. The the highest improvement is for Slovene and represents a $7.8 \%$ relative reduction in F-measure error on the complete task.

When features looking at the joint assignments of multiple words are added, the model achieves much larger improvements (models joint FS in the Table) across all languages. ${ }^{7}$ The highest overall improvement compared to the pipelined approach is again for Slovene and represents $22.6 \%$ reduction in error for the full task; the reduction is $40 \%$

\footnotetext{
${ }^{7}$ Since the optimization is stochastic, the results are averaged over four runs. The standard deviations are between 0.02 and 0.11 .
}

\begin{tabular}{|l|l|c|c|c|}
\hline Language & Model & Tag & Lem & T+L \\
\hline English & tag oracle & 100 & 98.9 & 98.7 \\
English & m-best oracle & 97.9 & 99.0 & 97.5 \\
\hline English & no tags & - & 94.3 & - \\
English & pipelined & 90.9 & 95.9 & 90.0 \\
English & local FS & 90.8 & 95.9 & 90.0 \\
English & joint FS & 91.7 & 96.1 & 91.0 \\
\hline \hline Bulgarian & tag oracle & 100 & 84.3 & 84.3 \\
Bulgarian & m-best oracle & 98.4 & 90.7 & 89.9 \\
\hline Bulgarian & no tags & - & 73.2 & - \\
Bulgarian & pipelined & 87.9 & 78.5 & 74.6 \\
Bulgarian & local FS & 88.9 & 79.2 & 75.8 \\
Bulgarian & joint FS & 89.5 & 81.0 & 77.8 \\
\hline \hline Slovene & tag oracle & 100 & 85.9 & 85.9 \\
Slovene & m-best oracle & 98.7 & 91.2 & 90.5 \\
\hline Slovene & no tags & - & 78.4 & - \\
Slovene & pipelined & 89.7 & 82.1 & 78.3 \\
Slovene & local FS & 90.8 & 82.7 & 80.0 \\
Slovene & joint FS & 92.4 & 85.5 & 83.2 \\
\hline \hline Czech & tag oracle & 100 & 83.2 & 83.2 \\
Czech & m-best oracle & 98.1 & 88.7 & 87.4 \\
\hline Czech & no tags & - & 78.7 & - \\
Czech & pipelined & 92.3 & 80.7 & 77.5 \\
Czech & local FS & 92.3 & 80.9 & 78.0 \\
Czech & joint FS & 93.7 & 83.0 & 80.5 \\
\hline
\end{tabular}

Table 4: Results on the test set achieved by joint and pipelined models and oracles. The numbers represent tag-set prediction F-measure (Tag), lemma-set prediction F-measure (Lem) and F-measure on predicting complete tag, lemma analysis sets $(\mathbf{T}+\mathbf{L})$.

relative to the upper bound achieved by the $m$-best oracle. The smallest overall improvement is for English, representing a 10\% error reduction overall, which is still respectable. The larger improvement for Slavic languages might be due to the fact that there are many more forms of a single lemma and joint reasoning allows us to pool information across the forms.

\section{Conclusion}

In this paper we concentrated on the task of morphological analysis, given a lexicon and unannotated data. We showed that the tasks of tag prediction and lemmatization are strongly dependent and that by building state-of-the art models for the two subtasks and performing joint inference we can improve performance on both tasks. The main contribution of our work was that we introduced a joint model for the two subtasks which incorporates dependencies between predictions for multiple word types. We described a set of features and an approximate inference procedure for a global log-linear model capturing such dependencies, and demonstrated its effectiveness on English and three Slavic languages.

\section{Acknowledgements}

We would like to thank Galen Andrew and Lucy Vanderwende for useful discussion relating to this work. 


\section{References}

Meni Adler, Yoav Goldberg, and Michael Elhadad. 2008 Unsupervised lexicon-based resolution of unknown words for full morpholological analysis. In Proceedings of $A C L$ 08: $H L T$.

Galen Andrew, Trond Grenager, and Christopher Manning. 2004. Verb sense and subcategorization: Using joint inference to improve performance on complementary tasks. In $E M N L P$.

Erwin Marsi Antal van den Bosch and Abdelhadi Soudi. 2007. Memory-based morphological analysis and partof-speech tagging of arabic. In Abdelhadi Soudi, Antal van den Bosch, and Gunter Neumann, editors, Arabic Computational Morphology Knowledge-based and Empirical Methods. Springer.

R. H. Baayen, R. Piepenbrock, and L. Gulikers. 1995. The CELEX lexical database.

Antal Van Den Bosch and Walter Daelemans. 1999. Memory-based morphological analysis. In Proceedings of the 37th Annual Meeting of the Association for Computational Linguistics.

Alexander Clark. 2002. Memory-based learning of morphology with stochastic transducers. In Proceedings of the 40th Annual Meeting of the Association for Computational Linguistics (ACL), pages 513-520.

Shay B. Cohen and Noah A. Smith. 2007. Joint morphological and syntactic disambiguation. In EMNLP.

Michael Collins. 2000. Discriminative reranking for natural language parsing. In $I C M L$.

M. Collins. 2002. Discriminative training methods for hidden markov models: Theory and experiments with perceptron algorithms. In EMNLP.

S. Cucerzan and D. Yarowsky. 2000. Language independent minimally supervised induction of lexical probabilities. In Proceedings of ACL 2000.

Markus Dreyer, Jason R. Smith, and Jason Eisner. 2008. Latent-variable modeling of string transductions with finite-state methods. In Proceedings of the Conference on Empirical Methods in Natural Language Processing (EMNLP), pages 1080-1089, Honolulu, October.

Tomaž Erjavec and Saǎo Džeroski. 2004. Machine learning of morphosyntactic structure: lemmatizing unknown Slovene words. Applied Artificial Intelligence, 18:1741.

Tomaž Erjavec. 2004. Multext-east version 3: Multilingual morphosyntactic specifications, lexicons and corpora. In Proceedings of LREC-04.

Jenny Rose Finkel, Christopher D. Manning, and Andrew Y. Ng. 2006. Solving the problem of cascading errors: Approximate bayesian inference for linguistic annotation pipelines. In $E M N L P$.

Nizar Habash and Owen Rambow. 2005. Arabic tokenization, part-of-speech tagging and morphological disambiguation in one fell swoop. In Proceedings of the 43rd Annual Meeting of the Association for Computational Linguistics.
Sittichai Jiampojamarn, Colin Cherry, and Grzegorz Kondrak. 2008. Joint processing and discriminative training for letter-to-phoneme conversion. In Proceedings of ACL08: HLT, pages 905-913, Columbus, Ohio, June.

M. Marcus, B. Santorini, and Marcinkiewicz. 1993. Building a large annotated coprus of english: the penn treebank. Computational Linguistics, 19.

Raymond J. Mooney and Mary Elaine Califf. 1995. Induction of first-order decision lists: Results on learning the past tense of english verbs. Journal of Artificial Intelligence Research, 3:1-24.

Eric Sven Ristad and Peter N. Yianilos. 1998. Learning string-edit distance. IEEE Transactions on Pattern Analysis and Machine Intelligence, 20(5):522-532.

Sunita Sarawagi and William Cohen. 2004. Semimarkov conditional random fields for information extraction. In $I C M L$.

Kristina Toutanova and Mark Johnson. 2008. A bayesian LDA-based model for semi-supervised part-of-speech tagging. In nips08.

Richard Wicentowski. 2002. Modeling and Learning Multilingual Inflectional Morphology in a Minimally Supervised Framework. Ph.D. thesis, Johns-Hopkins University.

R. Zens and H. Ney. 2004. Improvements in phrase-based statistical machine translation. In HLT-NAACL, pages 257-264, Boston, USA, May. 